\title{
Oxacillin plus ertapenem rapidly clears persistent left ventricular assist device-related methicillin-susceptible Staphylococcus aureus bacteremia
}

\author{
H Andrew Wilsey ${ }^{1}$, Barbara Pisani ${ }^{1}$, and Thomas O'Neill J ${ }^{1}$ \\ ${ }^{1}$ Wake Forest Baptist Medical Center
}

February 24, 2022

\begin{abstract}
There is an increasing use of left ventricular assist devices (LVADs) as bridge to transplantation or permanent destination therapy in the heart failure patient population. Infection remains a common complication in LVADs, with Gram-positive skin flora as predominant pathogens implicated, including Staphylococcus aureus. While there is emerging evidence for synergistic antibiotic combinations with methicillin resistant Staphylococcus aureus, there remains a significant gap in the literature for persistent methicillin susceptible Staphylococcus aureus (MSSA) bacteremia. In this article, we describe the first successful treatment of persistent LVAD-related bacteremia with salvage oxacillin plus ertapenem. The salvage therapy described here must be balanced by the risks for toxicity, impact on resistance, microbiota disruption, drug shortages, and patient costs. This combination warrants further evaluation in the clinical setting to better establish its role in our expanding patient population.
\end{abstract}

\section{Title}

Oxacillin plus ertapenem rapidly clears persistent left ventricular assist device-related methicillin-susceptible Staphylococcus aureus bacteremia

Authors:

H Andrew Wilsey, PharmD, BCCCP ${ }^{1}$; Barbara Pisani, DO²; Thomas J O'Neill IV, MD, PhD²;

1: Department of Pharmacy, Atrium Health Wake Forest Baptist, Winston Salem, NC

2: Division of Cardiology, Department of Internal Medicine, Atrium Health Wake Forest Baptist, Winston Salem, NC

Corresponding Author: H Andrew Wilsey, PharmD, BCCCP, 1 Medical Center Boulevard, Winston-Salem BC 27157 hwilsey@wakehealth.edu ; ORCID 0000-0003-3672-1929

Co-Author emails: bpisani@wakehealth.edu tjoneill@wakehealth.edu

Ethics and integrity statement: The authors have no conflicts of interest to declare. No funding was utilized for this manuscript. Patient was informed of risks and benefits of allowing their information to be used in this case report and provided signed consent.

\section{Abstract:}

There is an increasing use of left ventricular assist devices (LVADs) as bridge to transplantation or permanent destination therapy in the heart failure patient population. Infection remains a common complication in LVADs, with Gram-positive skin flora as predominant pathogens implicated, including Staphylococcus aureus. While there is emerging evidence for synergistic antibiotic combinations with methicillin resistant 
Staphylococcus aureus, there remains a significant gap in the literature for persistent methicillin susceptibleStaphylococcus aureus ( MSSA) bacteremia. In this article, we describe the first successful treatment of persistent LVAD-related bacteremia with salvage oxacillin plus ertapenem. The salvage therapy described here must be balanced by the risks for toxicity, impact on resistance, microbiota disruption, drug shortages, and patient costs. This combination warrants further evaluation in the clinical setting to better establish its role in our expanding patient population.

Key words

Bacteremia, LVAD infection, Staphylococcus aureus, combination therapy, oxacillin, ertapenem

Introduction

The management of end stage heart failure remains limited by donor availability and recipient candidacy, which has resulted in increasing use of mechanical circulatory support (MCS) as bridge to transplantation or permanent destination therapy. Left ventricular assist devices (LVADs) offload a failing left ventricle, enhancing patient survival and quality of life. As newer generations of LVADs continue to reduce thrombosis risk our MCS patient population will continue to expand. However, infection remains a significant complication, increasing a patient's morbidity and mortality. A patient's driveline is the percutaneous tube connecting the internal VAD pump and the extracorporeal controlled unit. The driveline renders a patient highly susceptible to microbial contamination and represents a common source of infection. For this reason, Gram-positive skin flora are commonly implicated in LVAD-associated infections, including Staphylococcus aureus ${ }^{1}$. The management of this patient population is guided by observational data and expert opinion and may be further complicated by pathogen type, infection location, and patient surgical/transplant candidacy status. While there is emerging data on synergistic antibiotic combinations for the management of methicillin resistant Staphylococcus aureus, there remains a significant gap in the literature for persistent methicillin sensitiveStaphylococcus aureus ( MSSA) ${ }^{2}$. There are a handful of both in-vitro and in-vivo studies that suggest a synergistic effect of cefazolin and ertapenem combination therapy for persistent MSSA ${ }^{3-5}$. Here we describe the successful use of oxacillin and ertapenem combination therapy in the treatment of LVAD-associated persistent MSSA bacteremia.

Case presentation

A 51-year-old male with history of ischemic cardiomyopathy had an LVAD HeartMate III implanted in November 2019. He developed his first VAD-specific infection in April 2020, further classified as lateonset $^{6}$. The patient's driveline drainage culture was positive for MSSA and was started on oral doxycycline 100mg every 12 hours which was continued as suppressive therapy. September 2021, patient was admitted with worsening driveline infection (DLI). During this admission, he underwent incision and drainage with excisional debridement of infected subcutaneous tissue. MSSA with the same susceptibility patterns as prior wound culture was isolated and after several days of intravenous cefazolin, patient was transitioned to oral doxycycline 100mg and oral rifampin 300mg every 12 hours. Rifampin treatment was anticipated for 6 weeks, however patient was unable to tolerate due to gastrointestinal adverse effects and stopped therapy at 4 weeks. The patient was readmitted four months following surgical debridement, found to have MSSA on blood cultures. Susceptibilities remained similar, with an increase from $<0.25$ to 0.5 MIC reported for oxacillin. He was started on cefazolin for one day, and then transitioned to oxacillin $2 \mathrm{~g}$ every 4 hours. Complicating care, patient was noted to have a small mass concerning for vegetation on his tricuspid valve and was pacemaker dependent. The patient remained clinically stable but with ongoing bacteremia despite 7 days of targeted therapy. Source control was hindered given patient was not a candidate for transplant, given his current smoking status and pacemaker dependency. Ultimately, the decision was made to add ertapenem $1 \mathrm{~g}$ every 24 hours to $12 \mathrm{~g}$ continuous infusion oxacillin for salvage therapy. Within 24 hours of starting combination therapy, patient had resolution of persistent bacteremia. He was kept on ertapenem $1 \mathrm{~g}$ every 24 hours for 7 days total and transitioned to intravenous oxacillin $12 \mathrm{~g}$ a day for 6 weeks. No adverse events were noted with the addition of ertapenem and remains without relapse for 3 months.

Discussion 
The successful management of persistent MSSA bacteremia is highly dependent on source control, which is a significant challenge in the LVAD patient population. Source control may only be achieved via a device exchange, device removal during orthotopic heart transplant or explantation. It is important to stress here that unlike other medical device-related infections, exchange of VADs for source control is generally avoided due to the complexity of the surgical procedure and concerns regarding risk of reinfection of newly implanted device. Patients who are a candidate for neither transplant, removal nor replacement must rely on medical management. Aminoglycosides are generally discouraged given lack of clear-cut efficacy and documented toxicity issues. Rifampin may be considered, but frequently not started until cultures clear to avoid development of resistance. In recent years, much attention has been given to the synergistic combination of beta-lactam antibiotics for the management for MRSA bacteremia ${ }^{2}$. However, few data exist for therapeutic options for persistent MSSA bacteremia.

Ulloa et al recently published the largest case series of 11 patients with persistent MSSA bacteremia who were successfully treated with salvage ertapenem and cefazolin ${ }^{4}$. The mean duration of bacteremia was 6 days (range, 4-9 days) on appropriate antibiotics. Interestingly, among the 9 cases where blood cultures were collected daily, bacteremia cleared within 24 hours of ertapenem in 8 cases (88\%). Even more so remarkable considering two of these cases were tricuspid valve endocarditis with $>2 \mathrm{~cm}$ vegetations. This robust response even without obtaining surgical source control appears to be mirrored in our patient case. Within in the LVAD population, there exists one published case series. Carenas-Alvarez et al. reported 2 cases of refractory MSSA bacteremia in LVAD recipients successfully treated with cefazolin plus ertapenem 3 . The first patient had ertapenem added to his therapy after 10 days of cefazolin and cleared cultures one day after starting salvage treatment. The second patient developed MSSA bacteremia through suppressive intravenous cefazolin and was therefore treated with intravenous oxacillin. However, after eight days of persistently positive cultures, switched to cefazolin with ertapenem with resolution of bacteremia within 24 hours.

While several studies have noted increased tolerability and even improved outcomes in patients receiving cefazolin as compared to anti staphylococcal penicillins (ASP), providers may have concerns regarding potential inferior cefazolin activity under high inoculum conditions ${ }^{4}$. The combination of ASP and ertapenem has even less data - the largest published in late 2021. El-Dalati describe eight patients treated with ASP (oxacillin or nafcillin) and ertapenem. Combination treatment was initiated following 5 days of persistent bacteremia. Blood sterility was achieved in all patients; six patients had documented clearance within one day ${ }^{8}$. Here, oxacillin plus ertapenem was the most common regimen, suggesting that salvage combination therapy may be used with either ASP or cefazolin. This conclusion is strengthened in our patient case. To the best of our knowledge, this is the first report describing use of an ASP based salvage regimen in LVAD-associated bacteremia.

In nearly all cases, a rapid clearance of blood cultures was observed. The basis for this combination leverages differences in affinity for various protein binding proteins (PBPs). Beta-lactam antibiotics exert their bactericidal effects by irreversibly interfering with the enzymes involved in cell wall synthesis, PBPs. Inhibiting enzymatic activity results in impaired cell wall synthesis, cell wall destabilization, and subsequently cell death. Carbapenem antibiotics have a higher affinity for PBP1 as compared to antistaphylococcal betalactams. Therefore, their addition would complement the relative proclivity of cefazolin and ASP for PBP2. While oxacillin is thought to have higher affinity for PBP1, the data presented in this case report and by El-Dalati et al. suggest oxacillin as a suitable agent for this salvage therapy ${ }^{8}$. Oxacillin may exhibit a similar mechanism for synergy with complimentary PBP-2 binding or may possibly offer more complete PBP1 saturation.

This combination has repeatedly demonstrated a clear and rapid resolution of persistent bacteremia. This has serious consequences for the MCS patient population, as time to blood sterilization with S. aureus may be associated with improved clinical outcomes and lower rates of death ${ }^{9}$. However, there are certainly risks associated with this salvage therapy that warrant consideration. Virtually every antibiotic has been associated with Clostridioides difficile infection (CDI). Therefore, it is important to consider the risk-benefit of 
adding a secondary antimicrobial agent that may result in long-lasting changes to the human gut microbiota. Similarly, the introduction of antibiotics to our patient population invariably leads to resistance. Further investigation is warranted to assess the full impact of carbapenem use on patient and community resistance patterns. Questions remain regarding the choice between ASP as compared to cefazolin in the setting of risk of acute kidney injury versus efficacy with inoculum conditions. Lastly and perhaps most importantly, further research is needed to capture whether rapid blood sterilization offers a patient-centered outcome benefit.

\section{Future directions and conclusion}

In conclusion, we present a case of the successful use of oxacillin and ertapenem for the management of persistent MSSA bacteremia in an LVAD patient. Staphylococcus aureus is notorious for the common metastatic spread of infection, which has been correlated with the duration of bacteremia. Prompt clearance of blood cultures was noted within 24 hours from combination initiation, similarly to available published data. Based on in vitro data, cefazolin had been utilized previously for salvage therapy. This is the first case report in the MCS setting describing successful use of oxacillin with ertapenem. The salvage therapy described here must be balanced by the risks for toxicity, impact on resistance, microbiota disruption, drug shortages, and patient costs. Questions regarding optimal timing of initiation of ertapenem, duration of combination therapy, repeated efficacy of combination therapy, and extrapolation to other MCS patients in which source control may be challenging. It is also critical to recognize that no patient-centered benefit has been estab-

lished and combination therapy risks have not been fully explored. Ultimately, this combination warrants further evaluation in the clinical setting to better establish its role in patient care.

\section{References}

1. Kusne S, Mooney M, Danziger-Isakov L, et al. An ISHLT consensus document for prevention and management strategies for mechanical circulatory support infection. J Heart Lung Transplant . 2017;36(10):11371153. doi:10.1016/j.healun.2017.06.007

2. Wilsey HA, Burgess DR, Burgess DS. Focusing the Lens on the CAMERA Concepts: Early Combination $\beta$-Lactam and Vancomycin Therapy in Methicillin-Resistant Staphylococcus aureus Bacteremia. Antimicrob Agents Chemother . 2020;64(7):e00360-20. doi:10.1128/AAC.00360-20

3. Cardenas-Alvarez JL, Suarez J, Motoa G, et al. Cefazolin plus ertapenem and heart transplantation as salvage therapy for refractory LVAD infection due to methicillin-susceptible Staphylococcus aureus: A case series. J Card Surg . 2021;36(12):4786-4788. doi:10.1111/jocs.16040

4. Ulloa ER, Singh KV, Geriak M, et al. Cefazolin and Ertapenem Salvage Therapy Rapidly Clears Persistent Methicillin-Susceptible Staphylococcus aureus Bacteremia. Clin Infect Dis Off Publ Infect Dis Soc Am . 2019;71(6):1413-1418. doi:10.1093/cid/ciz995

5. Sakoulas G, Olson J, Yim J, Singh NB, Kumaraswamy M, Quach DT, Rybak MJ, Pogliano J, Nizet V. Cefazolin and Ertapenem, a Synergistic Combination Used To Clear Persistent Staphylococcus aureus Bacteremia. Antimicrob Agents Chemother. 2016 Oct 21;60(11):6609-6618. doi: 10.1128/AAC.01192-16.

6. Hannan MM, Husain S, Mattner F, et al. Working formulation for the standardization of definitions of infections in patients using ventricular assist devices. J Heart Lung Transplant . 2011;30(4):375-384. doi:10.1016/j.healun.2011.01.717

7. Qu Y, Peleg AY, McGiffin D. Ventricular Assist Device-Specific Infections. J Clin Med . 2021;10(3):453. doi:10.3390/jcm10030453

8. El-Dalati S, Sridaran S, Uricchio M, Kline EG, Shields R. Oxacillin plus ertapenem combination therapy leads to rapid blood culture clearance and positive outcomes among patients with persistent MSSA bacteraemia: a case series. JAC-Antimicrob Resist . 2021;3(3):dlab148. doi:10.1093/jacamr/dlab148 
9. Defining persistent Staphylococcus aureus bacteraemia: secondary analysis of a prospective cohort study - ScienceDirect. Accessed January 5, 2022. https://www.sciencedirect.com/science/article/pii/S1473309920304473?via\%3Dihub 